\title{
Diagnostic Yield of Bronchoscopic Techniques in Evaluating Primary Lung Cancer: The Philippine General Hospital (PGH) Experience
}

\author{
Michelle Anne M. Encinas-Latoy, MD, ${ }^{1}$ Marvin C. Masalunga, MD, ${ }^{2}$ \\ Roland Reuben B. Angeles, MD $^{3}$ and Anna Katrina G. Tojino, MD ${ }^{3}$ \\ ${ }^{1}$ Department of Pathology, College of Medicine, University of the Philippines Manila \\ ${ }^{2}$ Department of Laboratories, Philippine General Hospital, University of the Philippines Manila \\ ${ }^{3}$ Section of Pulmonary Medicine, Department of Medicine, Philippine General Hospital, University of the Philippines Manila
}

\begin{abstract}
Objectives. To determine the overall diagnostic yield of bronchoscopy-guided sampling methods in detecting lung cancer at the University of the Philippines, Philippine General Hospital. The diagnostic yield, equivalent to sensitivity, is defined as the number of bronchoscopic sampling or biopsy procedures with a diagnosis of malignancy divided by the total number of confirmed malignant cases.
\end{abstract}

Methods. This is a cross-sectional, retrospective sensitivity study involving bronchoscopy procedures from January 2014 to December 2018. Surgical Pathology and Cytology Reports of eligible cases were accessed through the institutional Laboratory Information System. Sensitive patient information was omitted, and each case was assigned a unique code. The overall diagnostic yield/sensitivity of bronchoscopy and the diagnostic yield/sensitivity of each technique were calculated.

Results. A total of 100 patients satisfied the inclusion and exclusion criteria. Primary lung malignancies are more common in males and the elderly. The most common primary lung cancer is adenocarcinoma (33\%). Bronchoscopy, regardless of whether single or multiple techniques were used, has a diagnostic yield of $86 \%$ (Cl: $77.6-92.1 \%)$. Of the individual techniques, those that obtain solid tissues (endobronchial and transbronchial biopsies; $88.2 \%$ [Cl: 78.1-94.8\%] and 80.0\% [Cl: 28.4-99.5\%], respectively) have higher yields compared to techniques that obtain cytologic samples (bronchial washing and brushing; 54.2\% [43.7-64.4\%] and 70.1\% [58.6-80\%], respectively).

Conclusion. Bronchoscopy, as a diagnostic procedure for pulmonary malignancies, has relatively high sensitivity and may be used for lesions located centrally and can be inspected visually. A multidisciplinary approach to patient selection for bronchoscopy helps improve the utility of the various bronchoscopic techniques.

Key Words: Lung Neoplasms, Bronchoscopy, Cytodiagnosis, Surgical Pathology

\section{INTRODUCTION}

Fiberoptic bronchoscopy (FOB) is an endoscopic procedure that allows visualization of the airways for diagnostic and therapeutic purposes. ${ }^{1}$ It is safe, convenient to perform, and well tolerated by patients, even as an outpatient procedure. Sampling procedures that make use of a fiberoptic bronchoscope include bronchial washings (BW), bronchial brushings (BB), endobronchial biopsies (EBB), transbronchial needle aspiration (TBNA), and

Corresponding author: Michelle Anne M. Encinas-Latoy, MD Department of Pathology

College of Medicine

University of the Philippines Manila

547 Pedro Gil St., Ermita, Manila 1000, Philippines

Email: mmencinaslatoy@up.edu.ph bronchoalveolar lavage (BAL).

The diagnostic yield of FOB depends on several factors, including the location of the lesion (higher yield in central than peripheral lesions), the expertise, and the facilities available in a medical center. ${ }^{1}$ In a study by Ghazarian et 
al., the diagnostic yield of EBB in patients with a peripheral endobronchial lesion visualized on FOB was $81.1 \%{ }^{2}$ The study by Labbe et al. showed that the overall sensitivity of non-guided bronchoscopy was $25.6 \%$, while sensitivities for bronchial aspiration, BAL, and BB were $14.2 \%, 11.6 \%$, and $16.5 \%$, respectively. ${ }^{3}$ Ost et al. reported a diagnostic yield of $53.7 \%$ (312 of 581 patients). The same study reported that transbronchial biopsy (TBB) was diagnostic in $43.2 \%$, TBNA in $47.4 \%, \mathrm{BB}$ in $37.8 \%$, and BAL in $19.3 \%{ }^{4}$ In another study, the diagnostic yields of BW, EBB, and BB are $28.3 \%, 77.5 \%$, and $53.7 \%$ of patients with visible lesions, respectively. ${ }^{5}$ In all studies, the yield is higher for techniques that obtain solid tissue samples, e.g., TBB and EBB, compared to other techniques.

FOB has been in use for many years at the University of the Philippines - Philippine General Hospital (UPPGH) to evaluate pulmonary masses. However, PubMed database searches reveal very few local studies that have discussed the yield of bronchoscopic techniques in the pathologic diagnosis of lung cancer. ${ }^{6,7}$ An unpublished local study by Palma and Jorge (2018) reported that of 32 outpatient diagnostic procedures in 2016-2017, 26 employed bronchoscopy techniques to evaluate pulmonary masses. ${ }^{8}$ For inpatients, 108 of 270 underwent bronchoscopy for the same purpose. However, the study did not mention the diagnostic yield of bronchoscopy and instead focused on other factors associated with the diagnosis of pulmonary malignancies.

In this study, the diagnostic yield of bronchoscopic techniques in evaluating lung cancer, defined as the number of bronchoscopic sampling or biopsy procedures with a diagnosis of malignancy divided by the total number of malignant cases confirmed on biopsy or cytology, was investigated. ${ }^{1}$ Specifically, the study aimed to determine the individual and overall diagnostic yield of bronchoscopyguided sampling methods in detecting primary lung cancer, irrespective of the sampling method that established the final diagnosis.

\section{MATERIALS AND METHODS}

This is a cross-sectional, retrospective, diagnostic sensitivity study involving all patients who underwent bronchoscopy at the Philippine General Hospital, from January 2014 to December 2018, with a final histopathologic diagnosis of primary lung cancer. The definitive histopathologic diagnosis should have been established either by bronchoscopy or other procedures, such as CT-guided aspiration biopsy, resection, open thoracotomy, excision biopsy, and thoracoscopic biopsy. Before the conduct of the study, ethical clearance was secured from the University of the Philippines - Manila Research Ethics Board (UPMREB).

\section{Sample Size and Inclusion/Exclusion Criteria}

The method by Buderer (1996) was used to get the target sample size. ${ }^{9}$ To estimate an expected diagnostic sensitivity of at least $80 \%$, with a $95 \%$ confidence interval width of $10 \%$, and prevalence of at least $90 \%$, at least 70 cases needed to be included.

The criteria for inclusion were 1) presence of a pulmonary mass; 2) FOB performed with biopsy or sampling of the pulmonary mass using any bronchoscopic technique (BB, BW, EBB, TBB, TBNA, and BAL); 3) a final pathologic diagnosis of lung cancer established by bronchoscopy or another diagnostic procedure, including non-bronchoscopic procedures and definitive surgical management; and 4) the bronchoscopy procedure should have been done anytime from January 2014 to December 2018 at the Philippine General Hospital. Cases with unconfirmed cytology or surgical pathology results (e.g., "atypical cells suspicious for malignancy" with no follow-up studies) and cases of metastatic lung disease were excluded from the study.

\section{Data Collection, Processing, and Analyses}

Surgical Pathology and Cytology Reports accessible through the Laboratory Information System of PGH were reviewed. All patient identifiers and sensitive data were omitted during data processing. Individual patient records were assigned their unique code identifiers in the data collection forms.

All bronchoscopic results that show lung cancer were considered as true positives (TP). If bronchoscopy failed to reveal a specific diagnosis, but other methods, including resection procedures, eventually diagnosed lung cancer, the subject was considered a false negative (FN).

Diagnostic yield or sensitivity of bronchoscopy for primary lung cancer is defined as $\mathrm{TP} /(\mathrm{TP}+\mathrm{FN})$. For the overall diagnostic yield/sensitivity of bronchoscopy, all cases fulfilling the inclusion criteria were used, irrespective of the sampling method or specific bronchoscopy technique. For the study's secondary objective, the diagnostic yield/ sensitivity of each technique was calculated separately.

\section{RESULTS}

\section{Patient Demographics}

Four hundred twenty-eight (428) bronchoscopy procedure records from 2014 to 2018 were reviewed. Of these, 100 patients fulfilled the inclusion/exclusion criteria and were thus included in the study. The patients' characteristics are summarized in Table 1, which shows that primary lung malignancies are more common in males than in females, and in the elderly.

Of the 428 cases, 21 had an unresolved or unconfirmed diagnosis of pulmonary malignancy and were thus excluded. Twelve were females, and nine were males, with ages of 45 to 81 . In 12 of the 21 cases, immunohistochemistry studies to confirm malignancy diagnosis were requested by the Pathologist. In comparison, in nine cases, the specimens submitted for cytology or surgical pathology were inadequate. In all 21 cases, no records of immunohistochemistry studies 
Table 1. Patient characteristics of the 100 cases included in the study

\begin{tabular}{lr}
\hline Sex & \\
Male & $70(70 \%)$ \\
Female & $30(30 \%)$ \\
\hline Age & \\
$35-44$ & $4(4 \%)$ \\
$45-54$ & $12(12 \%)$ \\
$55-64$ & $32(32 \%)$ \\
$65-74$ & $39(39 \%)$ \\
$75-84$ & $11(11 \%)$ \\
85 and above & $2(2 \%)$ \\
\hline
\end{tabular}

Table 2. Pathologic diagnoses of patients who underwent bronchoscopic procedures from January 2014 to December 2018

\begin{tabular}{lcc}
\multicolumn{1}{c}{ Diagnosis } & N & $\%$ \\
\hline $\begin{array}{l}\text { Adenocarcinoma/non-small cell carcinoma, } \\
\text { favor adenocarcinoma }\end{array}$ & 33 & $33 \%$ \\
\hline $\begin{array}{l}\text { Non-small cell carcinoma, not otherwise } \\
\text { specified/unclassified }\end{array}$ & 29 & $29 \%$ \\
\hline $\begin{array}{l}\text { Squamous cell carcinoma/non-small cell } \\
\text { carcinoma, favor squamous cell carcinoma }\end{array}$ & 26 & $26 \%$ \\
\hline $\begin{array}{l}\text { Small cell carcinoma/malignant round cell } \\
\text { neoplasm, favor small cell carcinoma }\end{array}$ & 11 & $11 \%$ \\
\hline Mucoepidermoid carcinoma & 1 & $1 \%$ \\
\hline
\end{tabular}

Table 3. Methods used to establish a final diagnosis of primary pulmonary malignancy

\begin{tabular}{lrr} 
Final Diagnostic Method & $\mathbf{n}$ & \% \\
\hline Bronchoscopy & 86 & $86 \%$ \\
CT-GAB* of lung mass & 8 & $8 \%$ \\
US-GAB** of lung mass & 1 & $1 \%$ \\
Regional lymph nodes biopsy & 1 & $1 \%$ \\
Cervical LN biopsy & 2 & $2 \%$ \\
VATS*** with lung biopsy & 1 & $1 \%$ \\
Thoracentesis of malignant effusion & 1 & $1 \%$ \\
\hline
\end{tabular}

${ }^{*}$ CT-GAB - CT-guided aspiration biopsy

** US-GAB - Ultrasound-guided aspiration biopsy

*** VATS - Video-assisted thoracoscopic surgery

or a repeat biopsy to confirm malignancy diagnosis were found. The rest of the bronchoscopy cases had diagnoses of a benign or non-neoplastic etiology.
The diagnoses and number of patients for each diagnosis are presented in Table 2. The most common diagnosis is adenocarcinoma, with 33\%. Representative images of some of these malignancies are shown in Figure 1.

\section{Diagnostic Yield of Bronchoscopic Procedures}

Although there are only 100 unique individuals included in this study, most of them underwent more than one technique per bronchoscopic procedure; for instance, patients who underwent BW also underwent BB. Therefore, the overall diagnostic yield of bronchoscopy was calculated on a per-patient basis, i.e., for any bronchoscopy procedure that utilizes at least two techniques, such as a combination of $\mathrm{BB}$ and $\mathrm{BW}$, even if only one technique yielded a positive result, the entire procedure was considered diagnostic. On the other hand, each specific technique's diagnostic yield was evaluated individually, vis-à-vis the final diagnostic method that yielded a malignancy diagnosis, which may be the bronchoscopic technique itself.

Of the 100 patients, 96 underwent bronchial washing (BW), 77 underwent bronchial brushing (BB), 68 underwent endobronchial biopsy (EBB), five underwent transbronchial biopsy (TBB), and one underwent bronchoalveolar lavage (BAL). Taken as a sum of the individual techniques, the overall diagnostic yield of bronchoscopy is $69.23 \%$ (171/247); however, the diagnostic yield of bronchoscopy for malignancy increases to $86 \%$ (Table 3), when each bronchoscopy procedure is considered as one case, even if more than one technique was employed.

Of the five bronchoscopic techniques, three were performed in at least sixty patients, either singly or in combination with other techniques: BW, BB, and EBB. Of these three techniques, EBB has the highest diagnostic yield at $88.2 \%$ (Table 4). The diagnostic yields of TBB and BAL are $80 \%$ and $100 \%$, respectively; however, the sample sizes for the latter two techniques are small (five and one, respectively).

\section{DISCUSSION}

The advent of new techniques such as navigational bronchoscopy and endobronchial ultrasound has improved

Table 4. Bronchoscopic techniques and diagnostic yield for primary lung cancer

\begin{tabular}{|c|c|c|c|c|c|c|}
\hline \multirow{2}{*}{ Diagnosis } & \multicolumn{6}{|c|}{ 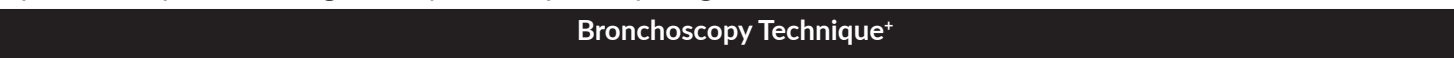 } \\
\hline & BW & BB & EBB & TBB & BAL & Any Technique* \\
\hline Benign & 11 & 6 & 3 & 0 & 0 & 7 \\
\hline Malignant & 52 & 54 & 60 & 4 & 1 & 86 \\
\hline Non-Diagnostic & 33 & 17 & 5 & 1 & 0 & 7 \\
\hline Total & 96 & 77 & 68 & 5 & 1 & 100 \\
\hline $\begin{array}{c}\text { Diagnostic yield } \\
\text { (\%) }\end{array}$ & $\begin{array}{c}54.2 \\
(\mathrm{Cl}: 43.7-64.4 \%)\end{array}$ & $\begin{array}{c}70.1 \\
\text { (Cl: } 58.6-80 \%)\end{array}$ & $\begin{array}{c}88.2 \\
\text { (Cl: 78.1-94.8\%) }\end{array}$ & $\begin{array}{c}80.0 \\
\text { (Cl: 28.4-99.5\%) }\end{array}$ & $\begin{array}{c}100 \\
\text { (Cl: } 2.5-100 \%)\end{array}$ & $\begin{array}{c}86 \\
\text { (Cl: } 77.6-92.1 \%)\end{array}$ \\
\hline
\end{tabular}

+BW - bronchial washing; BB - bronchial brushing; EBB - endobronchial biopsy; TBB - transbronchial biopsy; BAL - bronchoalveolar lavage

*This represents the over-all diagnostic yield of bronchoscopy; i.e., a diagnosis of malignancy was obtained regardless of the number of techniques done per bronchoscopic procedure. 

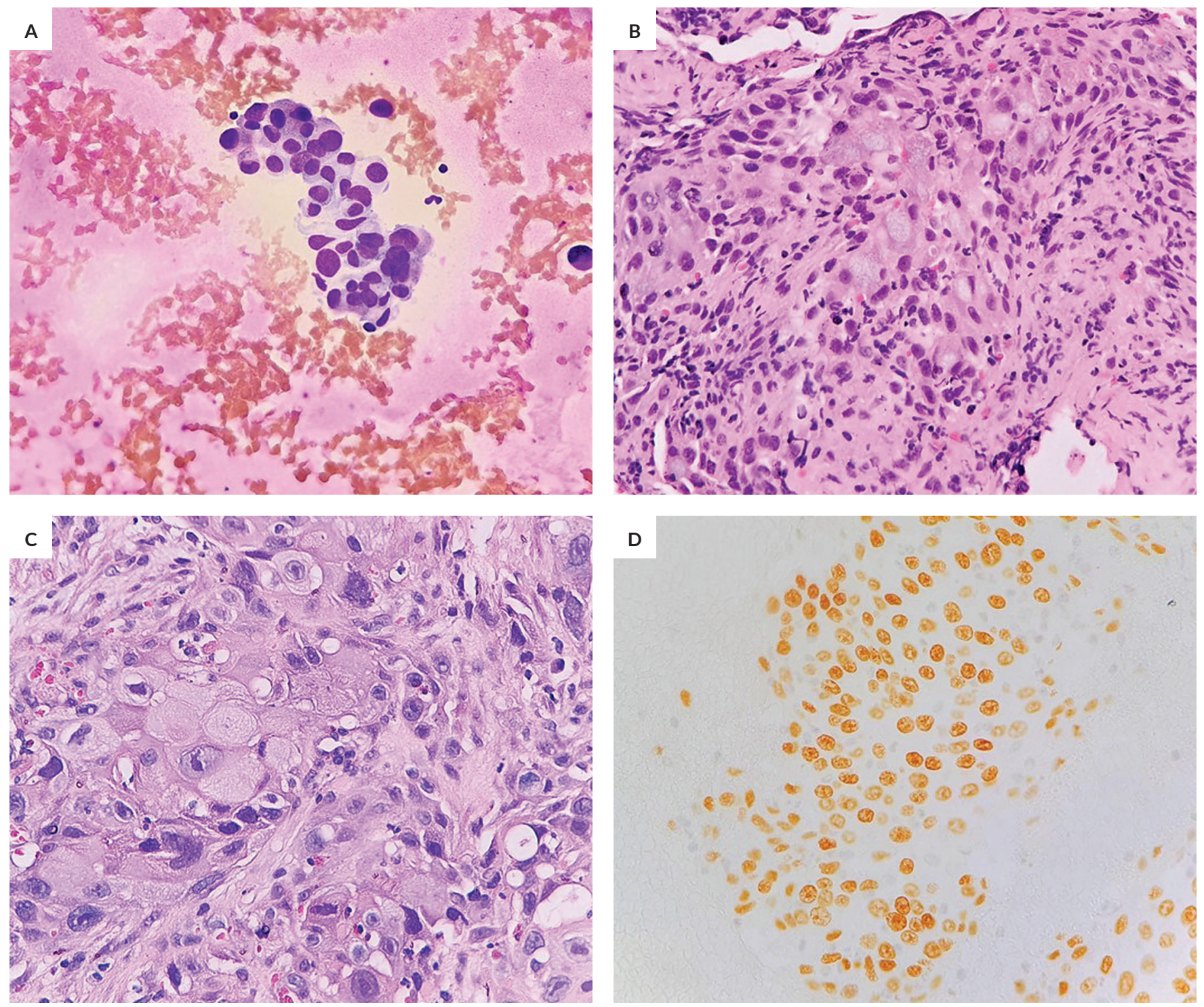

Figure 1. Lung malignancy specimens obtained via bronchoscopy. (A) Photomicrograph from a bronchial washing which yielded clusters of malignant cells, compatible with adenocarcinoma (DiffQuik stain, 400x). (B) Adenocarcinoma on tissue biopsy. Atypical and pleomorphic cells with hyperchromatic nuclei, interspersed within a fibrocollagenous stroma, appear to form vague, acinar structures with occasional globules of mucin (Hematoxylin and eosin stain, 400x). (C) Squamous cell carcinoma on tissue biopsy. Sheets of highly atypical and pleomorphic cells with irregular nuclei, prominent nucleoli, abundant cytoplasm, and foci of keratinization are seen (Hematoxylin and eosin stain, 400x). (D) For difficult cases, immunostains may be used, such as p40, which presents with strong, nuclear staining. p40 is used to confirm squamous cell carcinoma (immunohistochemistry with horseradish peroxidase, 400x).

the success rate of bronchoscopy in diagnosing even small peripheral lung lesions. ${ }^{10}$ In the University of the Philippines - Philippine General Hospital (UP-PGH), clinicians have access to fluoroscopic-guided bronchoscopy. This technique uses an ultra-thin scope, allowing access to more than just endobronchial lesions. The current practice in the Section of Pulmonary Medicine is that for patients presenting with suspicious nodules or masses, they are initially evaluated by a multidisciplinary team composed of specialists from the Pulmonary Medicine and Thoracic Surgery services to determine the best diagnostic approach, whether surgical, bronchoscopic, or image-guided.

In this study, the overall diagnostic yield of bronchoscopic techniques in evaluating primary lung cancer is $86 \%$. This includes all bronchoscopic techniques, regardless of whether the lesion is central or peripheral, or if an endobronchial lesion is visible or not. This diagnostic yield is high, comparable to published studies, particularly by Ghazarian et al., wherein the bronchoscopy diagnostic yield was $81.1 \% .^{2}$ 
A possible explanation for this relatively high sensitivity is the excellent system of patient selection for a bronchoscopy. Institutional practices in $\mathrm{PGH}$ include a preliminary evaluation with CT scan-based imaging, in which lesions are deemed accessible by bronchoscopy techniques if they have the following features: a more central location, at least 1 to $2 \mathrm{~cm}$ in size, and located near an airway, i.e., the CT-bronchus sign. Lesions that are more peripheral and without clear access via the airways are referred to the Section of Interventional Radiology for possible imageguided biopsy. BW, BAL, and $\mathrm{BB}$ are considered if the lesion appears diffuse or infiltrative. The fluoroscopy-guided biopsy is employed for the diagnosis of peripheral lung lesions with no visible endobronchial lesions. ${ }^{11}$

For lung lesions inaccessible by these techniques and highly suspected to be malignant, surgical biopsy via lobectomy or segmentectomy is considered. Other diagnostic methods aside from bronchoscopy, including CT scan and ultrasound, may be employed if bronchoscopy cannot establish the diagnosis. In this study, these imagingguided aspiration biopsy techniques demonstrated the final diagnosis in $9 \%$ of the cases. Other techniques used to confirm a malignancy are lymph node biopsies, videoassisted thoracoscopic surgery with biopsy, and thoracentesis of malignant effusion.

In terms of each bronchoscopic technique's diagnostic yield, EBB has the highest yield among the procedures done in at least 60 patients, at $88.2 \%$ (60/68). Previous international studies report the same finding of $\mathrm{EBB}$ giving a high yield. BW has the lowest yield at 54.2\% (52/96); nevertheless, this value is relatively higher than the study by Girard et al., wherein bronchial cytology techniques (e.g., aspiration or washing) reported a sensitivity of only $14.7 \%{ }^{12}$ The reason for this is in our setting, bronchial cytology is often still performed, even if the lesion is visible, contributing to its relatively high yield. In low resource settings without access to advanced bronchoscopic technology, such as endobronchial ultrasound guidance and ultra-thin bronchoscopes, bronchial cytology may still be employed. In an unpublished retrospective review by King et al., the diagnostic yield for malignancy was found to be adequate in 5 out of 20 specimens obtained via either technique washing or brushing. ${ }^{13}$ There were even samples wherein the result was positive for malignancy in the cytology samples, and yet was negative for the biopsy sample obtained during a single procedure. Therefore, despite their relatively low sensitivity, there remains some degree of utility for BW and BB, as they are relatively safe, easy to accomplish, and may be a valuable adjunct to endobronchial biopsy.

While this study aimed to give insight into the diagnostic yield of the various bronchoscopic techniques, certain limitations must be emphasized. The study included only malignant lesions confirmed by surgical pathology or cytology. Since this is a retrospective study, patients who were lost to follow-up before a definitive pathologic diagnosis of malignancy was made might have reduced the numbers that could have been diagnosed with each technique. The relationship between diagnostic yield and practice pattern variations among physicians (i.e., choice of specific bronchoscopy techniques used) was also not analyzed in this study.

No stringent criteria are being utilized in our institution to determine the best approach in a patient with a pulmonary mass. In a multidisciplinary setting composed primarily of pulmonologists, surgeons, oncologists, radiologists, and pathologists, clinical judgment, patient factors, and availability of resources all play a crucial role in determining the best diagnostic approach for any patient.

\section{CONCLUSION}

Bronchoscopy is a relatively sensitive method in detecting primary lung malignancies, especially when multiple techniques are employed in a given case. Of the various techniques available, those that obtain solid tissue samples, such as EBB, result in higher yields. An institutional patient selection criteria and a multidisciplinary approach also result in a higher diagnostic yield for a bronchoscopy.

\section{Recommendations}

The investigators of this study recommend the following as related future studies:

1. Relationship between clinical practice variations among physicians, i.e., case selection and specific bronchoscopy technique, and diagnostic yield in evaluating pulmonary malignancies.

2. Diagnostic yield of bronchoscopy in evaluating all pulmonary lesions, whether benign or malignant.

3. A similar study but with a prospective design and possibly involving multiple centers. Such studies may be designed to include a more thorough assessment of a lesion's clinical features, such as its exact location and size.

\section{Statement of Authorship}

MAMEL - Conceptualization and design of work; Interpretation of data; Drafting of Results and Discussion; Final approval of the version to be published.

MCM - Conceptualization and design of work; Acquisition and analysis of data; Drafting of Results and Discussion; Final approval of the version to be published.

RRBA and AKGT - Conceptualization of work; Analysis of data; Drafting of Discussion; Final approval of the version to be published.

\section{Author Disclosure}

All authors declared no conflicts of interest.

\section{Funding Source}

None. 


\section{REFERENCES}

1. Herth FJF. Bronchoscopic techniques in diagnosis and staging of lung cancer. Breathe. 2011; 7(4):325-37. doi: 10.1183/20734735.021510.

2. Ghazarian Z, Alziadat M, Sekhon R, Hanna M, Pandya T, Ismail M. Diagnostic yield of bronchoscopic lung biopsy in evaluating lung cancer. Chest. 2016 Oct; 150(4):S710A. doi: 10.1016/j.chest. 2016.08.805.

3. Labbé C, Beaudoin S, Martel S, Delage A, Joubert P, Drapeau C, et al. Diagnostic yield of non-guided flexible bronchoscopy for peripheral pulmonary neoplasia. Thorac Cancer. 2015 Jul; 6(4): 517-23. doi: 10.1111/1759-7714.12223

4. Ost DE, Ernst A, Lei X, Kovitz KL, Benzaquen S, Diaz-Mendoza $\mathrm{J}$, et al. Diagnostic yield and complications of bronchoscopy for peripheral lung lesions. Results of the AQuIRE Registry. Am J Respir Crit Care Med. 2016 Jan; 193(1):68-77. doi: 10.1164/ rccm.201507-1332OC.

5. Liam CK, Pang YK, Poosparajah S. Diagnostic yield of flexible bronchoscopic procedures in lung cancer patients according to tumor location. Singapore Med J. 2007 Jul; 48(7):625-31.

6. Bagano M, Aranas JC, Zanoria M. Lung cancer detection rate of conventional bronchoscopic techniques: A tertiary hospital experience June 2011 - December 2016. J Thorac Oncol. 2017 Nov; 12(11):S2164. doi: 10.1016./j.jtho.2017.09.1346.

7. Ponte FAF, Bisnar REM, Rivera MKR, Chavez CL. Endobronchial ultrasound-guided transbronchial needle aspiration in the Philippines: A preliminary retrospective cohort study on diagnostic performance. Philippine Journal of Chest Diseases. 2016 Jul-Sep; 17(3):13-9.
8. Palma M, Jorge M. Turnabout time and clinical factors associated with diagnosis of lung cancer at Philippine General Hospital, Manila, 2018. Unpublished.

9. Buderer NM. Statistical methodology: I. Incorporating the prevalence of disease into the sample size calculation for sensitivity and specificity. Acad Emerg Med. 1996 Sep; 3(9):895-900. doi: 10.1111/ j.1553-2712.1996.tb03538.x.

10. Khan KA, Nardelli P, Jaeger A, O'Shea C, Cantillon-Murphy P, Kennedy MP. Navigational bronchoscopy for early lung cancer: A road to therapy. Adv Ther. 2016 Apr; 33(4):580-96. doi:10.1007/ s12325-016-0319-4.

11. Barrios MJ, Senitko M, Majid A, Folch E, Bussy SF. Transbronchial biopsy with or without fluoroscopy? EC Pulmonology and Respiratory Medicine. 2019; 8(4):345-55.

12. Girard P, Caliandro R, Seguin-Givelet A, Lenoir S, Gossot D, Validire P, et al. Sensitivity of cytology specimens from bronchial aspirate or washing during bronchoscopy in the diagnosis of lung malignancies: an update. Clin Lung Cancer. 2017 Sep;18(5):512-8. doi: 10.1016/j.cllc.2016.11.009.

13. King R, Angeles R, Barbon C, Chua A, Ignacio J, Benedicto J. Barriers to timely lung cancer diagnosis and treatment in a tertiary hospital in the Philippines, 2020. Unpublished.
Have you read the current trends in Medical and Health Research in the Philippines?

\section{Acta Medica Philippina The National Health Science Journal}

\author{
Access Online: www.actamedicaphilippina.upm.edu.ph
}

